\title{
Defect study of Zn-doped $p$-type gallium antimonide using positron lifetime spectroscopy
}

\author{
C. C. Ling, S. Fung, and C. D. Beling \\ Department of Physics, The University of Hong Kong, Pokfulam Road, Hong Kong, China \\ Weng Huimin \\ Department of Modern Physics, University of Science and Technology of China, Hefei, China
}

(Received 2 February 2001; published 5 July 2001)

\begin{abstract}
Defects in $p$-type Zn-doped liquid-encapsulated Czochralski-grown GaSb were studied by the positron lifetime technique. The lifetime measurements were performed on the as-grown sample at temperature varying from $15 \mathrm{~K}$ to $297 \mathrm{~K}$. A positron trapping center having a characteristic lifetime of 317 ps was identified as the neutral $V_{\mathrm{Ga}}$-related defect. Its concentration in the as-grown sample was found to be in the range of $10^{17}-10^{18} \mathrm{~cm}^{-3}$. At an annealing temperature of $300^{\circ} \mathrm{C}$, the $V_{\mathrm{Ga}}$-related defect began annealing out and a new defect capable of trapping positrons was formed. This newly formed defect, having a lifetime value of $379 \mathrm{ps,}$ is attributed to a vacancy-Zn-defect complex. This defect started annealing out at a temperature of $580{ }^{\circ} \mathrm{C}$. A positron shallow trap having binding energy and concentration of $75 \mathrm{meV}$ and $10^{18} \mathrm{~cm}^{-3}$, respectively, was also observed in the as-grown sample. This shallow trap is attributed to positrons forming hydrogenlike Rydberg states with the ionized dopant acceptor $\mathrm{Zn}$.
\end{abstract}

DOI: 10.1103/PhysRevB.64.075201

PACS number(s): 61.72.Ji, 78.70.Bj

\section{INTRODUCTION}

Gallium antimonide $(\mathrm{GaSb})$ is a III-V semiconductor having a narrow band gap, small effective electron mass, and high electron mobility. GaSb is the basic material for a variety of lattice-matched optoelectronic devices working in the wavelength range of $0.8-4.3 \mu \mathrm{m}$. Because of the recent development of low loss optical fibers in the $2-4 \mu \mathrm{m}$ range, the research activity of $\mathrm{GaSb}$ has been enhanced by the need for compatible new optoelectronic materials. Reviews of the physics, the growth, and the device fabrication of the material GaSb can be found by Milines and Polyakov ${ }^{1}$ and Dutta and Bhat. ${ }^{2}$

Although understanding the defects in the material is crucial to device fabrication, very little was known about defects in GaSb. $V_{\mathrm{Ga}} G a_{\mathrm{Sb}}$ is an important defect in $\mathrm{GaSb}$ as it or its related complex is suspected to be the residual acceptor associated with the $p$-type conduction of as-grown undoped $\mathrm{GaSb}^{3-5}$ This residual acceptor was related to a deficiency of $\mathrm{Sb}$ or an excess of Ga (Refs. 6-8) and was doubly ionized. ${ }^{9}$ After annealing undoped GaSb for several hours, however, Allégre and Avérous ${ }^{5}$ observed the creation of $V_{\mathrm{Sb}}$ caused by thermal out-diffusion of $\mathrm{Sb}$. This donor defect consequently compensated the residual acceptor. However, prolonged annealing of the sample for several tens of hours finally led to an increase in the hole concentration. These observations can be understood by the formation of the $V_{\mathrm{Ga}} G a_{\mathrm{Sb}}$ acceptor resulting from the reaction of a $\mathrm{Sb}$ vacancy with a neighboring $\mathrm{Ga}$ atom. The $V_{\mathrm{Ga}} G a_{\mathrm{Sb}}$ acceptor was also suggested to be related to the $777 \mathrm{meV}$ luminescence signal in the photoluminescence (PL) (Refs. 4 and 1013) and cathodoluminescence (CL) (Refs. 14-16) spectra of a variety $\mathrm{GaSb}$ materials.

The positron lifetime technique is a nondestructive defect probe with selective sensitivity towards neutral or negatively charged open volume defects. Positrons implanted into a solid annihilate from either the bulk delocalized state or the localized defect state in which the positron is trapped by an open volume defect (normally a vacancy or vacancy agglomerate), which presents a potential well to the positron. As positrons annihilating from different states have different annihilation rates, defects can be identified by their own characteristic positron lifetimes. The defect concentration, charge state, or ionization energy can often be obtained after analyzing the lifetime spectra. Positron lifetime spectroscopy has been extensively employed to identify and characterize various types of defects in various IIII-V semiconductors. ${ }^{17-25}$ Reviews of the application of positron annihilation spectroscopy (PAS) on the study of semiconductor defects can be found in Refs. 26-28.

Though a large amount of defect information in III-V semiconductors has been obtained from PAS, there are only very few positron annihilation spectroscopic studies that report on the gallium antimonide ${ }^{29,30}$ Using the positron lifetime technique, Mahony et al. ${ }^{29}$ observed a 300 ps positron trapping defect and attributed it to positrons annihilating in a monovacancy-type defect. Dannefaer et al. ${ }^{30}$ have performed lifetime measurements on Te-doped GaSb. A positron trapping center with a lifetime of $297 \mathrm{ps}$ was observed and the bulk lifetime value was found to be $253 \mathrm{ps}$. Puska et al. ${ }^{31}$ reported an experimental value of $260 \mathrm{ps}$ for the GaSb bulk lifetime. In the same article, the theoretical lifetime values of $V_{\mathrm{Ga}}, V_{\mathrm{Sb}}$, and the divacancy $V_{\mathrm{Sb}} V_{\mathrm{Ga}}$ were reported to be 287, 307, and $350 \mathrm{ps,} \mathrm{respectively.}$

In this paper, we have performed positron lifetime studies on $\mathrm{Zn}$-doped $p$-type $\mathrm{GaSb}$ in order to investigate the defects in the material. For the as-grown sample, positron lifetime spectra were collected at temperatures varying from $15 \mathrm{~K}$ to $297 \mathrm{~K}$. An open volume defect having a characteristic lifetime of 317 ps and a positron shallow trap have been observed, which we attribute to a Ga-vacancy-related defect and the ionized $\mathrm{Zn}$ acceptor, respectively. Isochronal annealing studies have also been performed on the sample up to a temperature of $580^{\circ} \mathrm{C}$. 


\section{EXPERIMENT}

The $1 \times 1 \mathrm{~cm}^{2}$ samples used were cut from a $\mathrm{Zn}$-doped liquid-encapsulated Czochralski-LEC grown GaSb wafer purchased from the MCP Wafer Technology, Ltd. The thickness of the wafer was $0.5 \mathrm{~mm}$. From a room-temperature Hall measurement, the hole concentration of the wafer was measured to be $3.28 \times 10^{18} \mathrm{~cm}^{-3}$. The samples were degreased with acetone and ethanol and were then rinsed by deionized water. Isochronal annealing of the sample was performed in a nitrogen-hydrogen $(80 \%-20 \%)$ forming gas atmosphere at temperatures up to $580^{\circ} \mathrm{C}$. After each 30 minute annealing, the sample was cooled slowly to room temperature while still remaining in the forming gas atmosphere. The positron source employed was $30 \mu \mathrm{Ci}{ }^{22} \mathrm{NaCl}$ encapsulated with kapton foil. The source foil was sandwiched by a pair of the samples under investigation. The sample ensemble was inserted into a $10 \mathrm{~K}$ closed cycle He fridge with a narrow tail. The lifetime spectrometer used in the experiment had a resolution of 235 ps. The lifetime measurements were performed with the sample in darkness. Each of the lifetime spectra contained $4 \times 10^{6}$ counts. A $14.21 \% 385$ ps source correction was made to each spectrum. To test the accuracy of the obtained source correction, annealing study was also performed with ${ }^{22} \mathrm{Na}$ directly deposited onto the samples and the results obtained found to be consistent with those employing the kapton foil encapsulated source.

\section{RESULTS AND ANALYSIS}

\section{A. Lifetime study of as-grown sample under variations of measuring temperature}

The normalized measured lifetime spectra $S(t)$ are indeed the linear combination of terms corresponding to the positron annihilating at different sites [i.e., $S(t)=\sum_{i} I_{i} \exp \left(-t / \tau_{i}\right)$ where $I_{i}$ and $\tau_{i}$ are the intensity and the characteristic lifetime of positron annihilating at defect site $i]$. Each of the spectra was analyzed by the source $\operatorname{code}^{32}$ POSITRONFIT which fits the spectrum data to the sum of exponential decays after subtracting the background and the source contribution. The average lifetime $\tau_{\mathrm{av}}$ of each of the spectrum can also be calculated by the equation $\tau_{\mathrm{av}}=\Sigma_{i} I_{i} \tau_{i}$.

For the as-grown $\mathrm{Zn}$-doped GaSb sample, two lifetime components were required to give good fits to the spectra taken at temperatures from $150 \mathrm{~K}$ to $297 \mathrm{~K}$. In the fitting, all the parameters were treated as free. In this measuring temperature range, the long lifetime component was observed to be temperature independent and to have a value of $\tau_{2}=318$ \pm 7 ps. In order to eliminate the correlation between the fitted parameters, the spectra taken in this temperature range were refitted by fixing the long lifetime $\tau_{2}$ at this value. For spectra measured below $150 \mathrm{~K}$, a single lifetime component fit can give a good description to the experimental data. All of the fitted results of the lifetime parameters (i.e., $\tau_{1}$ and $I_{2}$ ) are shown in Figs. 1(a) and 1(b). From the figure, the long lifetime intensity $I_{2}$ was found to decrease from about $52 \%$ at $297 \mathrm{~K}$ with decreasing temperature. This decrease of $I_{2}$ was observed to correspond with the increase of the fitted value of $\tau_{1}$. At temperatures below $150 \mathrm{~K}$, the long lifetime

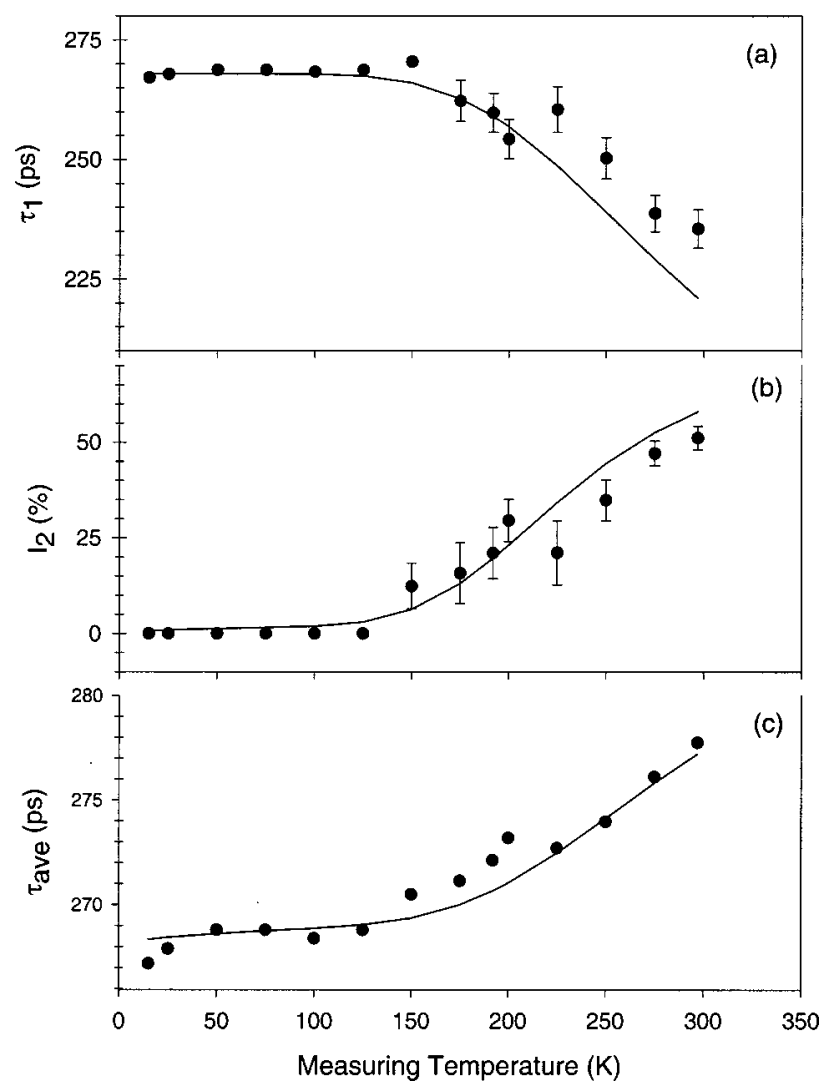

FIG. 1. Fitted positron lifetime parameters of the as-grown $\mathrm{Zn}$ doped GaSb sample as a function of the measuring temperature. The long lifetime component $\tau_{2}$ has constant value of $318 \pm 7$ ps. The solid curve was calculated from the model involving a vacancy trap and a shallow trap.

component completely disappeared (i.e., $I_{2}$ became zero) and the fitted value of $\tau_{1}$ saturated at about $268 \mathrm{ps}$. The average lifetime $\tau_{\mathrm{av}}$ as a function of the measuring temperature was also calculated and is shown in Fig. 1(c). The average lifetime decreases as a function of decreasing temperature and saturates at a value of $268 \pm 1$ ps as $T \leqslant 125 \mathrm{~K}$. The decrease of the average positron lifetime value with decreasing temperature is a direct result of the decrease in the long lifetime intensity $I_{2}$. As the long lifetime component is related to positron annihilation from positrons trapped in some open volume defect, these observations imply less annihilation events come from the vacancy-type trapped state as temperature decreases.

With the loss of the long lifetime component at measuring temperatures $T \leqslant 125 \mathrm{~K}$, a single lifetime model gives a good representation to the spectral data. This implies no positron trapping was observed in the sample in this low temperature range and thus $\tau_{1}$ becomes equal to the bulk lifetime. The ratio of the defect lifetime to the bulk lifetime has a value of 1.19 and thus the 318 ps component originates from a monovacancy defect. ${ }^{22}$ As the theoretical calculation shows that the $V_{\mathrm{Sb}}$ defect is positively charged for $p$-type material, ${ }^{33}$ the 318 ps component is most likely due to a $V_{\mathrm{Ga}}$-related defect. The observation that the $318 \mathrm{ps}$ component intensity drops with decreasing temperature can be ex- 
plained by the existence of a positron shallow trap in the sample bulk, which competes with the $V_{\mathrm{Ga}}$-related defect as a positron trap.

Positron shallow trapping and its thermal detrapping have been observed in electron-irradiated $\mathrm{GaAs}$ and $\mathrm{Si}$ in which the implanted thermalized positron forms hydrogenlike Rydberg states with negatively charged acceptors in these materials. ${ }^{34,35}$ As the shallow trapped positron experiences an electronic environment similar to that of the delocalized positron in the Bloch state, the positron lifetime value of the shallow trap is very close to that of the delocalized bulk state. The binding energy of a positron shallow trap (10 $\mathrm{meV}-100 \mathrm{meV}$ ) is less than that of a vacancy-type positron trap $(1 \mathrm{eV})$ and thus positron detrapping from the shallow trap is possible at or below room temperature. At low enough temperatures, detrapping becomes negligible and the positron essentially stays frozen on the negatively charged impurity. This implies more annihilation events originate from positrons in the shallow trap state and with less events contributed from the Ga vacancy deep trap. Therefore, the long lifetime intensity decreases. At temperatures lower than 150 $\mathrm{K}$, positrons annihilating from the shallow trap become dominant and only a single lifetime component is observed because the positron lifetime in the Rydberg state is very close to the positron bulk lifetime. The lifetime spectra of a system involving positron trapping into a vacancy-type deep trap and a shallow trap can be obtained by solving the rate equation of such a system. ${ }^{26}$ The lifetime spectra for such a system is a three-component exponential function [i.e., $\left.\sum_{i} I_{i} \exp \left(-t / \tau_{i}\right)\right]$ with lifetimes and intensities given as

$$
\tau_{1, \bmod }=\frac{2}{X+Y}, \quad \tau_{2, \bmod }=\frac{2}{X-Y}, \quad \tau_{3, \bmod }=\frac{1}{\lambda_{d}},
$$

$\lambda_{d}=\left(\tau_{d}\right)^{-1}$, where $\tau_{d}$ is the characteristic lifetime of the positron deep trap. $X$ and $Y$ are given by

$$
\begin{gathered}
X=\lambda_{b}+\kappa_{\mathrm{st}}+\kappa_{d}+\lambda_{\mathrm{st}}+\delta_{\mathrm{st}}, \\
Y=\sqrt{\left(\lambda_{b}+\kappa_{\mathrm{st}}+\kappa_{d}-\lambda_{\mathrm{st}}-\delta_{\mathrm{st}}\right)^{2}+4 \delta_{\mathrm{st}} \kappa_{\mathrm{st}}},
\end{gathered}
$$

where $\lambda_{b}$ and $\lambda_{\text {st }}$ are the positron annihilation rates of the bulk and the shallow trap, respectively. $\kappa_{d}$ is the trapping rate into the deep trap. $\kappa_{\text {st }}$ and $\delta_{\text {st }}$ are the trapping rate and the detrapping rate of the shallow trap, respectively. The trapping rate $\kappa$ is related to the equation $\kappa=\mu c$, where $\mu$ and $c$ are the specific trapping coefficient and the defect concentration, respectively. The component intensities are given by $^{26}$

$$
\begin{gathered}
I_{1, \bmod }=1-I_{2, \bmod }-I_{3, \bmod }, \\
I_{2, \bmod }=\frac{\delta_{\mathrm{st}}+\lambda_{\mathrm{st}}-\frac{1}{2}(X-Y)}{Y}\left[1+\frac{\kappa_{\mathrm{st}}}{\delta_{\mathrm{st}}+\lambda_{\mathrm{st}}-\frac{1}{2}(X-Y)}\right. \\
\left.+\frac{\kappa_{d}}{\lambda_{d}-\frac{1}{2}(X-Y)}\right],
\end{gathered}
$$

$$
I_{3, \bmod }=\frac{\kappa_{d}\left(\delta_{\mathrm{st}}+\lambda_{\mathrm{st}}-\lambda_{d}\right)}{\left[\lambda_{d}-\frac{1}{2}(X+Y)\right]\left[\lambda_{d}-\frac{1}{2}(X-Y)\right]} .
$$

As in practice it is difficult to decompose the lifetime spectra into the three components as shown above, only the longest lifetime $\tau_{3 \text {,mod }}$ can be well separated and the other two components (i.e., $\tau_{1 \text {, mod }}$ and $\tau_{2 \text {,mod }}$ ) merge together as one, giving ${ }^{36}$

$$
\begin{gathered}
\tau_{1}=\frac{I_{1, \mathrm{mod}}}{I_{1, \mathrm{mod}}+I_{2, \mathrm{mod}}} \tau_{1, \mathrm{mod}}+\frac{I_{2, \mathrm{mod}}}{I_{1, \mathrm{mod}}+I_{2, \mathrm{mod}}} \tau_{2, \mathrm{mod}}, \\
\tau_{2}=\tau_{3, \mathrm{mod}}, \\
I_{2}=I_{3, \mathrm{mod}}, \\
\tau_{\mathrm{av}}=I_{1, \mathrm{mod}} \tau_{1, \mathrm{mod}}+I_{2, \mathrm{mod}} \tau_{2, \mathrm{mod}}+I_{3, \mathrm{mod}} \tau_{3, \mathrm{mod}} .
\end{gathered}
$$

The relation between the trapping rate and detrapping of the shallow trap is given by ${ }^{37}$

$$
\delta_{\mathrm{st}}=\frac{\kappa_{\mathrm{st}}}{c_{\mathrm{st}}}\left(\frac{m k T}{2 \pi \hbar^{2}}\right)^{3 / 2} \exp \left(-\frac{E_{b}}{k T}\right)
$$

where $E_{b}$ is the binding energy of the positron shallow trap. The trapping rate of the shallow trap follows a temperature dependence of $\kappa_{\mathrm{st}} \sim T^{-0.5}{ }^{26}$ In order to model the experimental data, the lifetime values of bulk and shallow trap were fixed at $\tau_{b}=\tau_{\mathrm{st}}=268 \mathrm{ps}$ and the deep trap lifetime was taken as $\tau_{d}=318 \mathrm{ps}$. The experimental data of $\tau_{1}, I_{2}$, and $\tau_{\text {av }}$ shown in Fig. 1 were then fitted with Eqs. (1)-(5). Simultaneous good fits for all of the three experimental lifetime parameters $\tau_{1}, I_{2}$, and $\tau_{\text {av }}$ were obtained while employing a temperature-independent $\kappa_{d}$ assumption. The parameter values obtained for the best fit were $\mu_{\mathrm{st}}(15 \mathrm{~K})=10^{16} \mathrm{~s}^{-1}$, $c_{\mathrm{st}}=10^{18} \mathrm{~cm}^{-3}, \kappa_{d}=2 \times 10^{9} \mathrm{~s}^{-1}$, and $E_{b}=75 \mathrm{meV}$. The modeled $\tau_{1}, I_{2}$, and $\tau_{\text {av }}$ curves are plotted as a solid line in Fig. 1 and they are found to fit the experimental data well.

\section{B. Isochronal annealing lifetime study}

An isochronal annealing study on the sample was also performed up to a temperature of $580{ }^{\circ} \mathrm{C}$. After each of the annealings, positron lifetime measurements were conducted at room temperature. Similar to the as-grown sample measured at room temperature, all of these lifetime spectra could be well fitted by a two-component fitting. The fitted lifetime parameters and the average lifetime are shown in Fig. 2. The average lifetime is constant at about 274 ps for the as-grown, the $100^{\circ} \mathrm{C}$, and the $200^{\circ} \mathrm{C}$ annealed samples, and then drops with increasing annealing temperature. The average lifetime was found to be at about $271 \mathrm{ps}$ as the annealing temperature is between $400{ }^{\circ} \mathrm{C}$ and $500{ }^{\circ} \mathrm{C}$. A further reduction of the average lifetime to the value of about 266 ps was observed while the sample was annealed to $580{ }^{\circ} \mathrm{C}$. This behavior of $\tau_{\text {av }}$ reveals two annealing stages of two positron trapping defects, namely, beginning at around $300^{\circ} \mathrm{C}$ and $580^{\circ} \mathrm{C}$, re- 


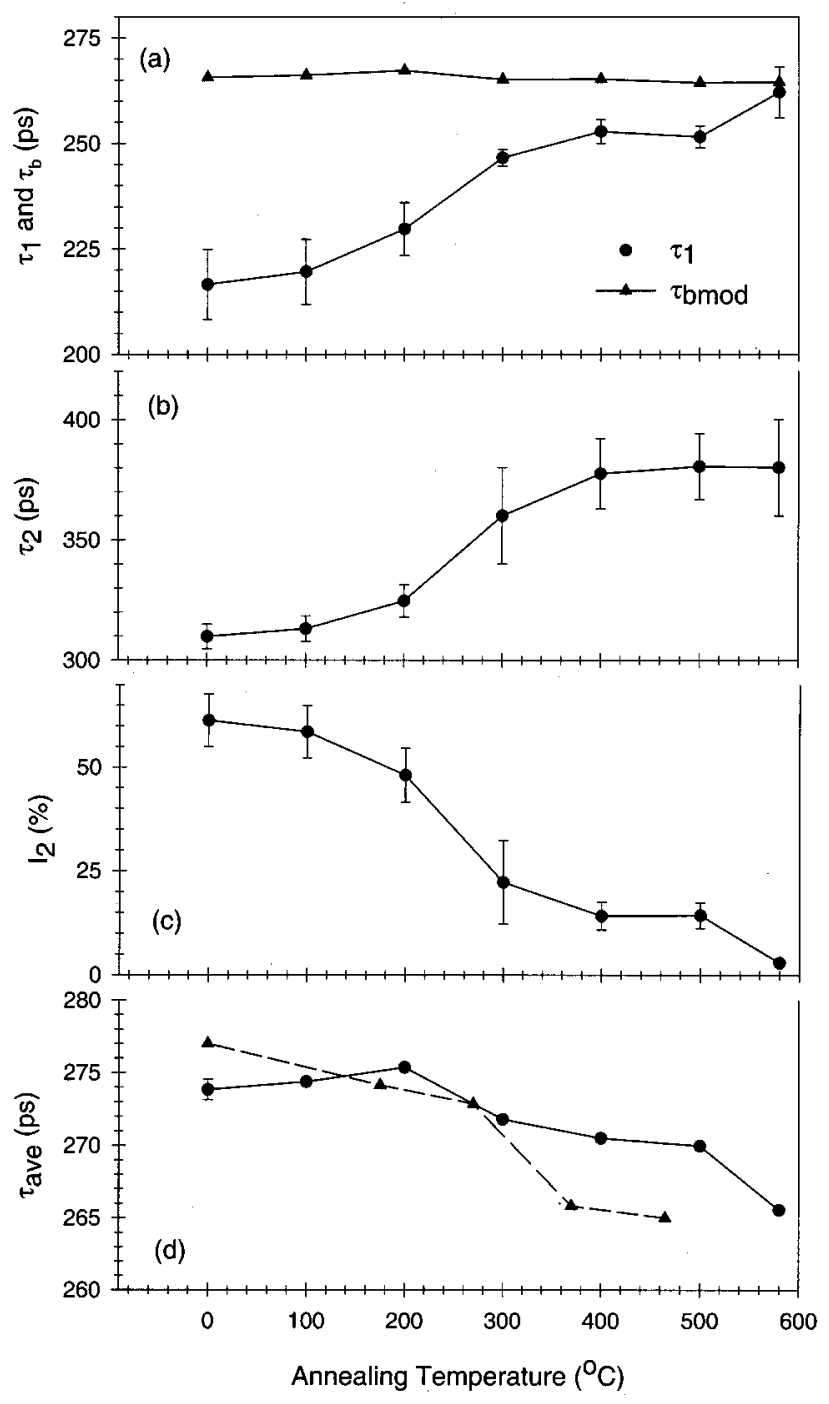

FIG. 2. Fitted lifetime parameters of the Zn-doped GaSb sample as a function of the annealing temperature are shown in circle and solid line. The two annealing stages are clearly seen at temperatures of $300{ }^{\circ} \mathrm{C}$ and $580{ }^{\circ} \mathrm{C}$. The $300^{\circ} \mathrm{C}$ annealing is related to the anneal out of the $V_{\mathrm{Ga}}$-related defect and the formation of a vacancy-type defect complex comprising the $\mathrm{Zn}$ dopant. The $580{ }^{\circ} \mathrm{C}$ annealing corresponds to the anneal out of this new defect. The average lifetime as a function of the annealing temperature for the undoped $\mathrm{GaSb}$ sample is shown as triangle and dotted line in (d).

spectively. Decomposition of the lifetime spectra further reveals the identify of the two defects.

Before the first annealing stage (i.e., $T_{\text {anneal }}<300^{\circ} \mathrm{C}$ ), the defect lifetime $\tau_{2}$ was found to be constant at about 316 $\pm 8 \mathrm{ps}$, which is statistical identical to the $V_{\mathrm{Ga}}$-related defect lifetime 318 ps observed on the as-grown sample as described in the previous section. At the annealing temperature of $300{ }^{\circ} \mathrm{C}$, the defect lifetime increases up to about $360 \mathrm{ps}$ and then saturates at about 379 ps while the annealing temperature is higher than or equal to $400^{\circ} \mathrm{C}$. As the roomtemperature Hall measurements for all of the annealed samples showed no movement of the Fermi level, any changes in the positron lifetime spectra must therefore be a consequence of the microstructural change of the positron trapping defect rather than the change of the specific positron trapping coefficient (which heavily depends on the defect charge state) induced by the movement of the Fermi level. This implies at the annealing temperature of $300{ }^{\circ} \mathrm{C}$ that the $V_{\mathrm{Ga}}$-related defect transformed to another kind of open volume defect also capable of trapping positrons and having a characteristic lifetime of 379 ps. For the second annealing stage at $580^{\circ} \mathrm{C}$, the decrease of $\tau_{\text {av }}$ is due to the drop of the fitted long lifetime intensity $I_{2}$, while there is no change in the defect lifetime value $\tau_{2}$. This implies that the newly formed defect started annealing out at the temperature of $580^{\circ} \mathrm{C}$.

In order to gain more information on the defect formed at $T_{\text {anneal }}=300^{\circ} \mathrm{C}$, an annealing positron lifetime study was performed on undoped $\mathrm{GaSb}$. The obtained spectra for the as-grown and low-temperature annealed samples were difficult to decompose. However, the average lifetime as a function of the annealing temperature is shown as dotted line and triangle in Fig. 2(c). From the figure, only a single annealing stage was found in the range $T_{\text {anneal }}=275^{\circ} \mathrm{C}-370^{\circ} \mathrm{C}$, which is close to the $300{ }^{\circ} \mathrm{C}$ anneal out found in the $\mathrm{Zn}$-doped sample, and thus is also attributed to the anneal out of $V_{\mathrm{Ga}}$-related defect. However, it is interesting to note, for the undoped sample, that the average lifetime drops to the bulk lifetime value, i.e., about $266 \mathrm{ps}$, for $T_{\text {anneal }} \geqslant 370^{\circ} \mathrm{C}$. This implies, unlike the $\mathrm{Zn}$-doped sample, that no new positron trapping defect was formed while the Ga vacancy anneals in the undoped sample. This leads us to suggest that the defect being annealed out in the Zn-doped sample at the first annealing stage $\left(300^{\circ} \mathrm{C}\right)$ is, as with the undoped sample, the $\mathrm{Ga}$ vacancy. However, in the $\mathrm{Zn}$-doped sample, a reaction involving the Ga vacancy and the $\mathrm{Zn}$ dopant occurs so as to make a more stable defect.

Puska et al. ${ }^{31}$ have calculated the lifetime values of GaSb bulk and $V_{\mathrm{Sb}} V_{\mathrm{Ga}}$ divacancy and obtained results of $257 \mathrm{ps}$ and $350 \mathrm{ps}$, respectively. This gives a theoretical estimation of $\tau_{V_{\mathrm{Sb}} V_{\mathrm{Ga}}} / \tau_{b} \sim 1.36$. The observed $379 \pm 17 \mathrm{ps}$ lifetime component (which has a $\tau_{d} / \tau_{b}$ ratio of 1.41) is thus quite consistent with the theoretical divacancy value. This implies the newly formed defect may possibly be a divacancy-type defect complexed with Zn dopant. However, we cannot exclude the possibility that the 379 ps positron trap is a monovacancy-type defect complexed with $\mathrm{Zn}$ dopant. Dlubek et al. ${ }^{38}$ in a positron lifetime study on heavily $\mathrm{Zn}$ doped $p$-type InP, identified the 325 ps positron trapping center as the $V_{P}$-Zn defect. In a later study, Alatalo et al. ${ }^{39}$ confirmed this suggestion by monitoring the core electron annihilation from the defect site. It is interesting to note, however, that although $V_{\mathrm{P}}$ is positively charged in $p$-type $\mathrm{InP}$ and its $\mathrm{Zn}$ complex is neutral or negatively charged. Second, the observed lifetime value $325 \mathrm{ps}$ is significantly larger than that of the monovacancy $V_{\mathrm{P}}\left(\tau_{V_{\mathrm{P}}}=263 \mathrm{ps}\right)$. In conclusion then, we cannot draw any firm conclusion on the exact identity of this newly formed vacancy-Zn-complex defect based solely on the present information available. We shall refer to it simply as $D$.

With the simple trapping model, the trapping rate into the defect and the bulk lifetime can be calculated by the equa- 


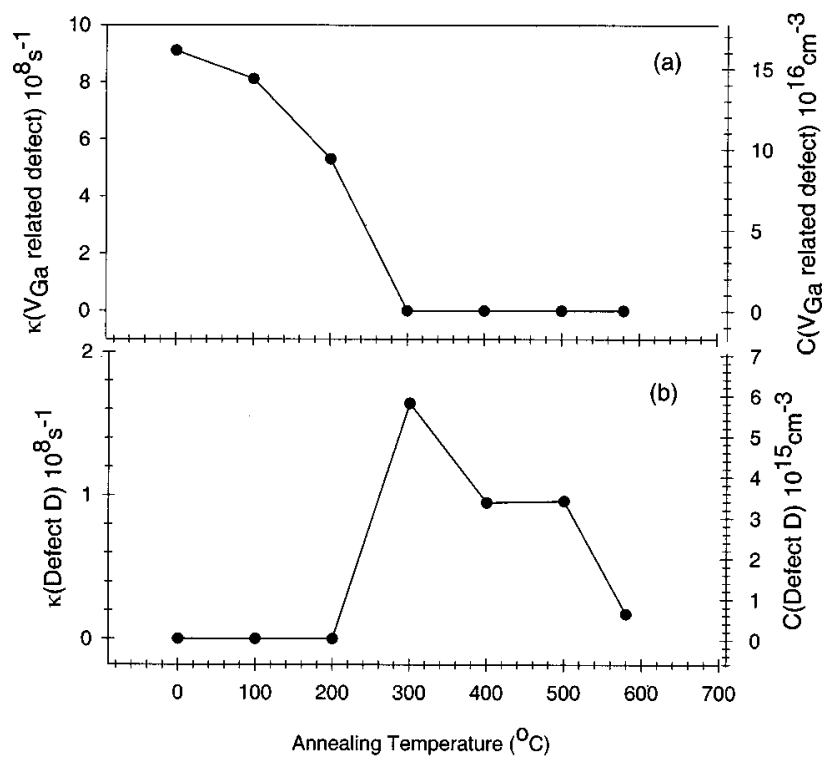

FIG. 3. (a) and (b) show the positron trapping rates of the $V_{\mathrm{Ga}}$-related defect and the vacancy-Zn complex having characteristic lifetime value of $379 \mathrm{ps}$ as a function of the annealing temperature, respectively. The defect concentrations were also calculated and are plotted at the right $y$ axis.

tions $\tau_{b \text {, mod }}=\left(I_{1} / \tau_{1}+I_{2} / \tau_{2}\right)^{-1}$ and $\kappa=\left(I_{2} / I_{1}\right)\left(\lambda_{b}-\lambda_{d}\right)$. The calculated $\tau_{b \text {,mod }}$ are shown in Fig. 2(a) and can be used to check the validity of the simple trapping model. ${ }^{26-28}$ The calculated $\tau_{b \text {, mod }}$ result shown in Fig. 2(a) suggests the simple trapping model is a valid one as $\tau_{b \text {, mod }}$ is found to have constant value of $266 \pm 1$ ps. This value is very close to that of $268 \pm 1$ ps obtained in the temperature-varying lifetime measurement on the as-grown sample.

The calculated trapping rates into the $V_{\mathrm{Ga}}$-related defect and the defect $D$ are shown in Figs. 3(a) and 3(b), respectively. The defect concentrations can also be deduced by the equation $\kappa=\mu c$ if the specific trapping coefficient of the defects are known. Although there are no available values on the specific positron trapping coefficients for the $V_{\mathrm{Ga}}$ and the 379 ps positron trapping defect $D$ in $\mathrm{GaSb}$, it is still of value to estimate the defect concentration by using the available data found in other semiconductors. As in Sec. III A, a simultaneous good fit to all of the three lifetime parameters was obtained only while assuming a temperature independent $\kappa_{V_{\mathrm{Ga}}}$. This implies that the $V_{\mathrm{Ga}}$ defect is neutral in charge because (i) the specific trapping rate of a neutral charge vacancy is temperature independent and (ii) that of a negatively charged vacancy follows the temperature dependence of $\mu \sim T^{-0.5}{ }^{41}$ Krause-Rehberg et al. ${ }^{40}$ have identified the $V_{\mathrm{Ga}}^{-}$defect in Ga-Al-Sb and its specific positron trapping coefficient was found to be $1 \pm 0.3 \times 10^{15} \mathrm{~s}^{-1}$ at $300 \mathrm{~K}$. For the case of $V_{\mathrm{Ga}}^{-}$in GaAs, Krause et al. ${ }^{21}$ obtained a value of $\mu(25 \mathrm{~K})=3 \times 10^{15} \mathrm{~s}^{-1}$, which implies a value of $\mu(300 \mathrm{~K})=9 \times 10^{14} \mathrm{~s}^{-1}$. This value is similar to that of $V_{\mathrm{Ga}}^{-}$in Ga-Al-Sb. ${ }^{40}$ From theoretical calculations, it was shown that the ratio between the specific trapping rate of a neutral vacancy and that of a negatively charged vacancy is about 1:5. ${ }^{41}$ Thus the specific trapping coefficient of the $\mathrm{Ga}$ vacancy of $\mathrm{GaSb}$ in the present study was taken as 2 $\times 10^{14} \mathrm{~s}^{-1}$.

For the case of the defect $D$, we have taken value of $\mu$ $=10^{15} \mathrm{~s}^{-1}$ to estimate the defect concentration as this value is the typical order of magnitude for neutral monovacancy or divacancy. The calculated concentration of $V_{\mathrm{Ga}}$ and the defect $D$ as a function of the annealing temperature are shown in Fig. 3. The concentration of the Ga vacancy is seen to be about $1.6 \times 10^{17} \mathrm{~cm}^{-3}$ for the as-grown sample. Its concentration decreases with increasing annealing temperature and finally anneals out at $300{ }^{\circ} \mathrm{C}$. For the case of the defect $D$, its concentration is below the detection limit for annealing temperatures below $300^{\circ} \mathrm{C}$. Formation of this defect was observed at an annealing temperature of $300{ }^{\circ} \mathrm{C}$ where its concentration reaches about $6 \times 10^{15} \mathrm{~cm}^{-3}$. This defect began annealing out at the temperature of $580^{\circ} \mathrm{C}$.

\section{DISCUSSION AND CONCLUSION}

In both our present studies, namely, the variation of measurement temperature and isochronal annealing, the bulk lifetime value of $\mathrm{GaSb}$ was found to be $267 \pm 1$ ps. Although there are only very few reports of positron lifetime studies on GaSb, experimental bulk lifetime values ${ }^{29-31}$ ranging from 253 ps to 260 ps have been previously reported. Our measured value here is about $4-5 \%$ larger than these reported values. One of the possibilities that may cause such a discrepancy is that in the present model analysis, we have assumed that the positron lifetime of the positron shallow trap is equal to that of the bulk because the electronic environments probed by a positron in the shallow trap state and that in the delocalized state are very similar. However, in the real situation, it may be possible that the lifetime of the shallow trap is a bit larger than that of the bulk, though they cannot be resolved in the spectral decomposition. This could in principle lead to a higher bulk lifetime value obtained from the model analysis. We have also performed room temperature lifetime measurements on an annealed undoped GaSb sample [as shown in Fig. 2(c)] and the results can clarify this uncertainty. For the undoped GaSb sample annealed at or above $370^{\circ} \mathrm{C}$, the average lifetime was found to be 266 ps and no positron trapping center was observed, which implies that the bulk lifetime value is $266 \mathrm{ps}$. As the concentrations of ionized acceptor (which are the main candidates for shallow traps) for the two samples (Zn-doped and undoped) are so different and the two obtained bulk lifetime values are effectively identical, it may be concluded that bulk lifetime value is $267 \mathrm{ps}$.

A positron trapping center having a lifetime of 317 ps has been observed at $T=150 \mathrm{~K}-297 \mathrm{~K}$ in the as-grown sample and also in the room-temperature measurements of the samples annealed below $300^{\circ} \mathrm{C}$. This defect was identified as either $V_{\mathrm{Ga}}$ or a $V_{\mathrm{Ga}}$-related defect because the ratio $\tau_{d} / \tau_{b}$ was found to be 1.19, which implies it is a monovacancy defect, and the $V_{\mathrm{Sb}}$ defect is positively charged for $p$-type material. According to the modeling in the previous section, this defect was found to be neutral in charge. The $\tau_{d} / \tau_{b}$ $\sim 1.19$ ratio observed hereby is also identical to the defect 
( $\tau_{d}=300 \mathrm{ps}$ and $\tau_{b}=253 \mathrm{ps}$ ) observed in the undoped GaSb samples reported by Mahony et al. ${ }^{29}$ though something like 5\% deviation exists in the exact lifetime value. In the study of Mahony et al., this defect was attributed to a neutral monovacancy-type defect. Puska et al. ${ }^{31}$ have calculated the positron lifetime of $V_{\mathrm{Ga}}$ to be 287 ps. The $317 \mathrm{ps}$ \pm 7 ps $V_{\mathrm{Ga}}$ lifetime value reported in the present study is larger than the value calculated by Puska et al. This is probably due to the defect relaxation that was not included in the calculation, or the change of the lifetime value resulting from the complex formation of $V_{\mathrm{Ga}}$. At the present stage, we are not able to give any conclusion on this issue.

The intensity of the $V_{\mathrm{Ga}}$-related lifetime component decreased with decreasing temperature and finally disappeared at $T \sim 125 \mathrm{~K}$. This observation has been explained by the existence of a positron shallow trap, which competes with the $V_{\mathrm{Ga}}$ defect in the positron trapping process. The positron trapping into the shallow trap becomes dominant at low temperatures as positron detrapping from it becomes negligible. Despite the fact that the positron trapping model involving a shallow trap and a vacancy can give an excellent description of the experimental data, another model that involves the charge state change of the $V_{\mathrm{Ga}}$-related defect induced by the lowering of the temperature can also explain the temperature dependence of the observed lifetime parameters. As temperature decreases, the Fermi level may possibly move closer to the valance band. The charge state of the $V_{\mathrm{Ga}}$-related defect may thus become positively charged and no longer trap positrons. As a result, positron trapping into the $V_{\mathrm{Ga}}$-related defect disappears at low temperature. In order to clarify the validity of this proposal, Hall measurements were performed on the as-grown sample at temperatures ranging from $6 \mathrm{~K}$ to $297 \mathrm{~K}$. The hole concentration of the $\mathrm{Zn}$-doped sample was found to be temperature independent with a value of about $(3.59 \pm 0.18) \times 10^{18} \mathrm{~cm}^{-3}$. This observation shows no evidence of significant Fermi level movement induced by temperature variation and thus this possibility does not seem to be the correct explanation for the lifetime parameter variation as a function of the measuring temperature.

The existence of $V_{\mathrm{Ga}}$-related defects in the present sample is consistent with the common viewpoint that $V_{\mathrm{Ga}} G a_{\mathrm{Sb}}$ is the residual acceptor found in most of the GaSb materials and is attributed to the $p$-type nature of undoped GaSb (Ref. 2 and references therein). Moreover, in PL and CL studies, a luminescence signal at about $777 \mathrm{meV}$ was observed in many independent studies and was related to the $V_{\mathrm{Ga}} G a_{\mathrm{Sb}}$ acceptor. ${ }^{4,14-16}$ However, it is worth pointing out that in the previous analysis, no account was taken of the partial occupancy of the Ga vacancy at different charge states induced by the thermal broadening of the Fermi distribution. For our present highly doped sample, the hole concentration measured by Hall measurement was found to be in the order of $10^{18} \mathrm{~cm}^{-3}$ in the whole temperature range of our experi- mentation. This concentration is as high as, or is larger than, the $N_{V}$ depending on the temperature of the material and thus the present sample is a degenerate semiconductor. With the use of the self-consistent semi-empirical tight binding model, $\mathrm{Xu}^{33}$ has obtained the energy levels (relative to the valance band) of $V_{\mathrm{Ga}}$ with charge states from +3 to -3 in GaSb as $0.028 \mathrm{eV}, 0.033 \mathrm{eV}, 0.041 \mathrm{eV}, 0.056 \mathrm{eV}$, and $0.091 \mathrm{eV}, 0.171$ $\mathrm{eV}$, and $0.291 \mathrm{eV}$, respectively. Assuming the Fermi level is at the bottom of the valence band, we can estimate the occupancies of $V_{\mathrm{Ga}}$ at different charge states from the equation $\left[V_{\mathrm{Ga}}^{Q}\right] /\left[V_{\mathrm{Ga}}^{Q+1}\right]=\left(g_{Q} / g_{Q+1}\right) \exp \left[-\left(E_{i}-E_{F}\right) / k T\right]$, where $E_{i}$ is the ionization energy for the process $V_{\mathrm{Ga}}^{Q} \rightleftharpoons V_{\mathrm{Ga}}^{Q+1}+e^{-}$. Taking the $g_{Q}$ to be all equal, at a temperature of $300 \mathrm{~K}$, the fractional occupancies of $\left[V_{\mathrm{Ga}}^{3+}\right],\left[V_{\mathrm{Ga}}^{2+}\right],\left[V_{\mathrm{Ga}}^{+}\right]$, and $\left[V_{\mathrm{Ga}}^{0}\right]$, were found to be $0.35,0.29,0.21$, and 0.12 , respectively, and the remaining Ga vacancies (about 3\%) to be in the negatively charged state. As most of the Ga vacancies are in the positively charged state (about $85 \%$ ), the $V_{\mathrm{Ga}}$ concentration calculated from the previous positron lifetime measurement were underestimated by a factor of $6.7(=1 / 0.15)$ because the positron lifetime technique is insensitive to the positively charged vacancy, which thus implies a Ga vacancy concentration of about $1 \times 10^{18} \mathrm{~cm}^{-3}$. Nevertheless, all of these estimated values of the $V_{\mathrm{Ga}}$ concentration are consistent with the expected residual acceptor concentration found in the asgrown GaSb material (Ref. 2 and the references therein).

The shallow trap in the as-grown sample was found to have a concentration of about $10^{18} \mathrm{~cm}^{-3}$. As the main acceptor in the present $\mathrm{Zn}$-doped sample is $\mathrm{Zn}$ impurity, it is reasonable to attribute this positron shallow trap to an ionized $\mathrm{Zn}$ acceptor. Furthermore, this statement is supported by the Hall measurement which gives the room temperature hole concentration of the present sample as 3.28 $\times 10^{18} \mathrm{~cm}^{-3}$. As the ionized acceptor concentration is equal to the hole concentration for the present heavily doped sample, the ionized $\mathrm{Zn}$ acceptor concentration was expected to be about $10^{18} \mathrm{~cm}^{-3}$, which is the same as the measured shallow trap concentration.

In the isochronal annealing study, at a temperature of $300{ }^{\circ} \mathrm{C}$, the $V_{\mathrm{Ga}}$-related defect began annealing out and a new defect capable of trapping positron was formed. This defect could be a vacancy or a divacancy-type defect complex related to the dopant $\mathrm{Zn}$. This defect was then observed to begin annealing out at a temperature of about $580^{\circ} \mathrm{C}$.

\section{ACKNOWLEDGMENTS}

This study was financially supported by the Research Grant Council, Hong Kong Special Administrative Region, China (Project No. HKU7134/99P) and the CRCG, The University of Hong Kong. The authors would like to thank W.K. Mui and M.K. Lui for their assistance while performing the measurements. 
${ }^{1}$ A.G. Milnes and A.Y. Polyakov, Solid-State Electron. 36, 803 (1993).

${ }^{2}$ P.S. Dutta and H.L. Bhat, J. Appl. Phys. 81, 5821 (1997).

${ }^{3}$ M.H. Van Maaren, J. Phys. Chem. Solids 27, 472 (1966).

${ }^{4}$ Y.J. Van Der Meulen, J. Phys. Chem. Solids 28, 25 (1967).

${ }^{5}$ J. Allégre and M. Avérous, in Defects and Radiation Effects in Semiconductors 1978, Institute of Physics Conference Series No. 46 (IOP, London, 1979), Chap. 5, p. 379.

${ }^{6}$ D. Effer and P.J. Effer, J. Phys. Chem. Solids 25, 451 (1964).

${ }^{7}$ E.J. Johnson I. Fillinski, and H.Y. Fan, in Proceedings of the 6th International Conference on Physics of Semiconductors, 1962, p. 375.

${ }^{8}$ M. D’Olne Campos, A. Gouskov, L. Gouskov, and J.C. Pons, J. Appl. Phys. 44, 2642 (1973).

${ }^{9}$ R.D. Baxter, R.T. Bate, and F.J. Reid, J. Phys. Chem. Solids 26, 41 (1965)

${ }^{10}$ M. Lee, D.J. Nicholas, K.E. Singer, and B. Hamilton, J. Appl. Phys. 59, 2895 (1986).

${ }^{11}$ Meng-Chyi Wu and Chi-Ching Chen, J. Appl. Phys. 72, 4275 (1992).

${ }^{12}$ A.Y. Polyakov, M. Stam, A.G. Milnes, R.G. Wilson, Z.Q. Fang, P. Rai-Choudhury, and R.J. Hillard, J. Appl. Phys. 72, 1316 (1992).

${ }^{13}$ P.S. Dutta, K.S.R. Koteswara Rao, H.L. Bhat, and V. Kumar, Appl. Phys. A: Mater. Sci. Process. 61, 149 (1995).

${ }^{14}$ P. Hidalgo, B. Méndez, P.S. Dutta, J. Piqueras, and E. Dieguez, Phys. Rev. B 57, 6479 (1998).

${ }^{15}$ G.N. Panin, P.S. Dutta, J. Piqueras, and E. Dieguez, Appl. Phys. Lett. 67, 3584 (1995).

${ }^{16}$ B. Méndez, P.S. Dutta, J. Piqueras, and E. Dieguez, Appl. Phys. Lett. 67, 2648 (1995).

${ }^{17}$ S. Dannefaer, T. Bretagnon, and D. Kerr, J. Appl. Phys. 80, 3750 (1996).

${ }^{18}$ K. Saarinen, P. Hautojärvi, P. Lanki, and C. Corbel, Phys. Rev. B 44, 10585 (1991)

${ }^{19}$ K. Saarinen, T. Laine, S. Kuisma, J. Nissilä, P. Hautojärvi, L. Dobrzynski, J.M. Baranowski, K. Pakula, R. Stepniewski, M. Wojdak, T. Suski, M. Leszcznski, I. Grzegory, and S. Porowski, Phys. Rev. Lett. 79, 3030 (1997).

${ }^{20}$ A. Polity and T. Engelbrecht, Phys. Rev. B 55, 10480 (1997).

${ }^{21}$ R. Krause, K. Saarinen, P. Hautojärvi, A. Polity, G. Gärtner, and C. Corbel, Phys. Rev. Lett. 65, 3329 (1990).
${ }^{22}$ G. Dlubek and R. Krause, Phys. Status Solidi A 102, 443 (1987).

${ }^{23}$ J. Mäkinen, T. Laine, K. Saarinen, P. Hautojärvi, C. Corbel, V.M. Airaksinen, and P. Gibart, Phys. Rev. Lett. 71, 3154 (1993).

${ }^{24}$ H.L. Au, C.C. Ling, P.K. Panda, T.C. Lee, C.D. Beling, and S. Fung, Phys. Rev. Lett. 73, 2732 (1994).

${ }^{25}$ Y.Y. Shan, C.C. Ling, A.H. Deng, B.K. Panda, C.D. Beling, and S. Fung, Phys. Rev. B 55, 7624 (1997).

${ }^{26}$ R. Krause-Rehberg and H.S. Leipner, Positron Annihilation in Semiconductors, Defect Studies, Vol. 127 of Springer Series in Solid-State Sciences (Springer-Verlag, Berlin, 1999).

${ }^{27}$ P. Hautojärvi and C. Corbel, in Positron Spectroscopy of Solids, Proceedings of the International School of Physics, Enrico Fermi, edited by A. Dupasquier and A.P. Mills, Jr. (ISO Press, Amsterdam, 1995), p. 491.

${ }^{28}$ M.J. Puska and R.M. Nieminen, Rev. Mod. Phys. 66, 841 (1994).

${ }^{29}$ J. Mahony, G. Tessaro, P. Mascher, H. Siethoef, and H.G. Brion, Mater. Sci. Forum 196-201, 1449 (1995).

${ }^{30}$ S. Dannefaer, W. Puff, and D. Kerr, Phys. Rev. B 55, 2182 (1997).

${ }^{31}$ M.J. Puska, S. Mäkinen, M. Manninen, and R.M. Nieminen, Phys. Rev. B 39, 7666 (1989).

${ }^{32}$ P. Kirkegaard, N.J. Pederson, and M. Eldrup (unpublished); P. Kirkegaard, M. Eldrup, O.E. Mogenson, and N.J. Pederson, Comput. Phys. Commun. 23, 307 (1981).

${ }^{33}$ H. Xu, J. Appl. Phys. 68, 4077 (1990).

${ }^{34}$ K. Saarinen, P. Hautojävi, A. Vehanen, R. Krause, and G. Dlubek, Phys. Rev. B 39, 5287 (1989).

${ }^{35}$ P. Mascher, S. Dannefaer, and D. Kerr, Phys. Rev. B 40, 11764 (1989).

${ }^{36}$ C.C. Ling, A.H. Deng, S. Fung, and C.D. Beling, Appl. Phys. A: Mater. Sci. Process. 70, 33 (2000).

${ }^{37}$ M. Manninen and R.M. Nieminen, Appl. Phys. A: Solids Surf. 26, 93 (1981).

${ }^{38}$ G. Dlubek, O. Brümmer, F. Plazaola, P. Hautojärvi, and K. Naukkarinen, Appl. Phys. Lett. 46, 1136 (1985).

${ }^{39}$ M. Alatoalo, H. Kauppinen, K. Saarinen, M.J. Puska, J. Mäkinen, P. Hautojärvi, and R.M. Nieminen, Phys. Rev. B 51, 4176 (1995).

${ }^{40}$ R. Krause-Rehberg, Th. Drost, A. Polity, G. Roos, G. Pensl, D. Volm, B.K. Meyer, G. Bischopink, and K.W. Benz, Phys. Rev. B 48, 11723 (1993).

${ }^{41}$ M.J. Puska, C. Corbel, and R.M. Nieminen, Phys. Rev. B 41, 9980 (1990). 